\title{
RAPID ON-CHIP SEPARATION OF PROTEINS AND IMMUNE COMPLEXES USING UV-INITIATED POLYACRYLAMIDE GELS
}

\author{
Amy E. Herr, Andrew A. Davenport†, Renée Shediac, Anup K. Singh \\ Biosystems Research, Sandia National Laboratories \\ Livermore, CA 94551-0969
}

\begin{abstract}
Proteomics seeks to identify proteins present during biological processes with the intent of characterizing important proteins and protein interactions. Recent advances in the application of microtechnology to biology have enabled development of increasingly complex microsystems that hold promise for proteomics, and clinical diagnostics, due to enabled rapid analysis of low abundance species. To that end, microdevice-based analysis of complex samples necessitates system architectures with localized functionality.

We have developed a cross-linked polyacrylamide gel photopolymerization process for the in situ fabrication of gel structures as a functional component of microfluidic-based proteomic and diagnostic systems. The straightforward fabrication process yields spatially localized gel matrices with the potential to increase functionality and improve performance of on-chip separation systems. In particular, our work involving UV-initiated polyacrylamide gels as a separation medium for sodium dodecyl sulfate polyacrylamide gel electrophoresis (SDS-PAGE) of proteins and electrophoretic immunoassays involving cytokines is described. The fabrication technique has enabled direct adaptation of slab gel SDS-PAGE of proteins to a chip-based format. Comparison of SDS-PAGE on-chip to slab gel has shown promising advantages, including separation times up to two orders of magnitude more rapid than those performed in gels $(30 \mathrm{~s} \mathrm{vs}$. $3600 \mathrm{~s})$ and separation distances that are a fraction of those required for slab SDS-PAGE (7 $\mathrm{mm} v s .50 \mathrm{~mm})$.
\end{abstract}

\section{INTRODUCTION}

Conventional SDS-PAGE is a time-consuming and laborintensive analytical technique conducted on a slab gel. Nevertheless, the technique is reliable, powerful, and yields valuable molecular weight information from complex samples. Coupled with isoelectric focusing, SDS-PAGE is considered the workhorse of proteomic analysis.[1] Much of the power of slab gel SDS-PAGE comes from the ability to tailor gel cross-linking to provide more or less sieving depending on the target sample (i.e., molecular weight, $M_{r}$, range of interest).

In the last decade, benchtop bioanalytical systems (slab gel and capillary-based) have been adapted for use in planar microfluidic format.[2,3,4,5] Use of microfluidic architectures has enabled rapid separations requiring short separation lengths. Exceptional performance improvements are attributed to the smallinjected analyte plug widths realizable using the microsystem format and the high applied electric field strengths attainable owing to the heat dissipating qualities of the microsystem. Further, microdevice formats are amenable to complex, integrated system designs and have increased potential for automation. The

$\uparrow$ Now at Dept. Mechanical Engineering Stanford University, Stanford CA 94305. aforementioned attributes of microfluidic systems make the approach promising for applications that require analysis of precious sample in a high throughput manner.

In an effort to leverage the strengths of sieving-type electrokinetic separations while addressing the limitations of macroscale analysis methods, researchers have employed polymeric structures for use in successful separations and analysis of DNA.[6,7] Even so, open channel zone electrophoresis (CZE) has been the method of choice for protein analysis. It is important to note that gel electrophoresis circumvents performance concerns and is superior to CZE in many respects, including: 1) high separation resolution due to low non-specific binding, 2) fast separations (seconds) using short length channels $(\mathrm{mm})$ due to the high surface area of the gel, 3) ready tailoring of gel porosity for specific applications, and 4) spatial-localization of photopatterned polyacrylamide (useful in multiplexing and integration).

While system development of the in situ photopolymerization process continues, results presented here indicate successful completion of a suite of protein and immune complex analyses employing the PAGE technique. Polymer matrices can be customized and localized using the straightforward procedure. Results presented demonstrate high separation resolution, throughput, and the ability to extract quantitative information for a variety of biologically relevant species.

\section{THEORY}

Apparent electrophoretic mobility and size differences between proteins enable differentiation of various species (for a detailed treatment of electrophoresis see Probstein[8]). Fractionation of analytes based on differences in both charge and size is the goal of SDS-PAGE using sieving media with pore sizes of between 0.5 and $3 \mathrm{~nm}$.[9] The ability to tailor the pore size of a cross-linked gel allows a system designer to optimize the separation media to resolve species within a size range of interest. Pore size is determined by the acrylamide monomer and bisacrylamide cross-linker concentrations in the initial unpolymerized mixture. An empirical relation has been developed for the separation behavior of SDS-protein complexes, as is relevant to SDS-PAGE. That separation relation has been determined to be a direct proportionality between the logarithm of the molecular weight and the mobility of the protein.[10] This straightforward relationship makes SDS-PAGE a useful, near universal technique for sizing unknown proteins.

One area where an SDS-PAGE like sizing mechanism may be useful is in the detection and characterization of immune complexes. Figure 1 presents a schematic illustrating a direct or non-competitive immunoassay. Immunodiagnostics employing this approach employ either antibody or antigen probes (e.g. hepatitis). In this work, fluorescently-labeled antigen probes were mixed with antibody-containing sample. During an electrokinetic separation of the sample, the presence of antibody can be 
determined through the formation of a second (lower apparent mobility) peak corresponding to the immune complex. The peak area/height of that second peak can be used as a measure of antigen concentration.

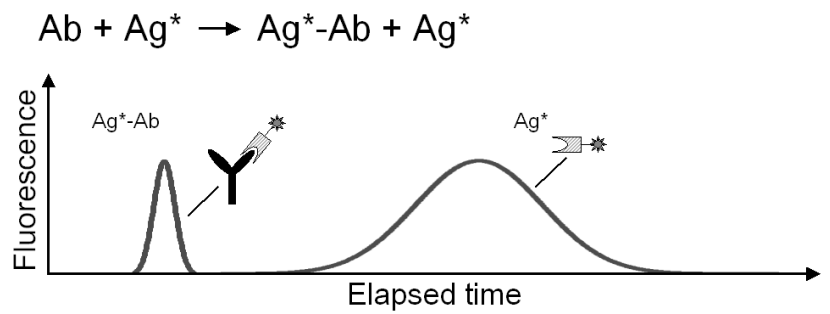

Figure 1. Schematic depiction of a direct immunoassay. Incubation of fluorescently labeled antigen $\left(\mathrm{Ag}^{*}\right)$ with unlabeled antibody (Ab) yields a fluorescent complex $\left(A g^{*}-A b\right)$ and excess $A g^{*}$. On-chip native PAGE is used to separate $\mathrm{Ag}^{*}$ from the complex, thus allowing detection and quantification of the immune complex.

To date, electrophoresis-based immunoassays have promised advantages over conventional immunoassay techniques. A capillary or microdevice-based electrophoretic immunoassay requires lower sample and reagent volumes, simultaneous determination of multiple analytes, rapid completion of immunoreaction, hence, rapid analysis, and direct detection of immune complex formation and dissociation, thus simplifying data interpretation.[11] As free solution electrophoresis separates species by differences in charge-to-mass ratio, the small degree of differentiation between the charge of antibody and immune complex make such separations difficult (i.e., in antigen-detecting systems). To address the difficulty, separations could be enhanced by either increasing electrophoretic mobility differences between antibody and immune complexes or by simply separating species based on relative size. The latter approach would benefit tremendously from tailoring of the pore size for high resolution in the antibody/immune complex molecular weight range.

\section{EXPERIMENTAL DETAILS}

Microchannel \& Photopatterned Cross-linked Gel Fabrication. Bioanalytical devices were fabricated in glass substrates as previously described[12] using standard photolithography, wet etching, and bonding techniques. The substrate was Schott D263 glass wafers (4-in. diameter, 1.1-mm thickness; S. I. Howard Glass Co.). The channels were $\sim 40 \mu \mathrm{m}$ deep $\mathrm{x} \sim 100 \mu \mathrm{m}$ wide with $6.7 \mathrm{~cm}$ long separation channels.

Prior to fabrication of the cross-linked gel, channels were functionalized with an acrylate-terminated self-assembled monolayer and subsequently coated with linear polyacrylamide using a two-step process similar to that described by Kirby et al.[13] The conditioning process enhances gel adhesion to the channel walls. Following the coating procedure, chips were flushed with deionized water for 5 minutes and stored filled with water at $5^{\circ} \mathrm{C}$.

Polyacrylamide gel structures were fabricated, using the reaction shown in Figure 2, to form sieving matrices for electrophoretic separations employing microfluidic devices. Sieving media composed of various gel concentrations were fabricated in situ using the four-step process depicted in Figure 3. Total gel concentration, T\% (monomer and cross-linker), was controlled by diluting a stock solution of $30 \%$ (37.5:1) acrylamide/bis-acrylamide (Sigma) with run buffer. The water- soluble photoinitiator 2,2'-azobis[2-methyl-N-(2hydroxyethyl)propionamide] (VA-086, Wako Chemicals) was added in a $5 \mathrm{mg} / \mathrm{mL}$ concentration to the diluted monomer and cross-linker solution. This low viscosity monomer solution was then introduced, via pressure-driven flow, into all open channels of the microfluidic device (Figure 3). Select regions were masked using material opaque to UV-light (e.g., electrical tape, Rubylith) and the entire device was exposed to a UV source $(\lambda=365 \mathrm{~nm}$, $4 \mathrm{~W})$ for 20 minutes. After the exposure step, all open channels were flushed with run buffer or water to remove unpolymerized acrylamide solution. As depicted in Figure 3, the UV exposure step resulted in localization of a cross-linked gel matrix in unmasked device regions. This process can be repeated to allow fabrication of gels having various porosities in different regions of a single device. During process development, voids or 'tears' have been observed forming in proximity to channel junctions upon application of an electric field. The tears may arise from stresses induced by electroosmotic flow in channels filled with a nonuniformly polymerized gel. Efforts to enhance gel robustness are currently underway in our lab.

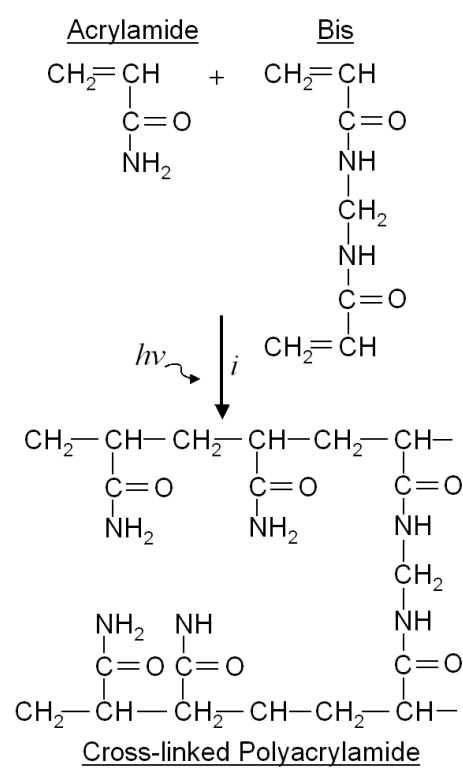

Figure 2. Polymerization reaction of acrylamide. Structural representations of acrylamide, $\quad N, N$ 'methylenebisacrylamide (Bis), and a segment of cross-linked polyacrylamide are shown. The initiator, in this case VA-086, is indicated as "i", while exposure to light is indicated by "hv". The concentration of acrylamide and Bis determine the pore size and cross-linking characteristics of the final gel structure. Schematic adapted from Chrambach and Rodbard [9].

Assays. Sample introduction and separation were performed using common electrokinetic injection methods.[14] The species transport was observed using standard epifluorescence microscopy techniques and digital image collection. Image analysis was conducted using available and in-house Java plug-ins developed using ImageJ (National Institutes of Health, Bethesda, MD, http://rsb.info.nih.gov/ij/). Images were corrected for background signal to adjust for spatial non-uniformities in the excitation and collection efficiencies.

For SDS-PAGE analysis, molecular weight markers (Sigma) consisted of five fluorescently labeled species in solution $(62 \mathrm{mM}$ Tris, $1 \mathrm{mM}$ EDTA, 3\% sucrose, $0.5 \%$ dithiothreitol, 2\% SDS and $0.005 \%$ bromphenol blue). The markers included: aprotinin (bovine lung), $M_{r} 6.5 \mathrm{kDa}$; $\alpha$-lactalbumin (bovine milk), $M_{r} 14.2$ $\mathrm{kDa}$; trypsin inhibitor (soybean), $M_{r} 20.1 \mathrm{kDa}$; carbonic anhydrase (bovine erythrocyte), $M_{r} 29 \mathrm{kDa}$; and alcohol dehydrogenase (horse liver), $M_{r} 39 \mathrm{kDa}$ were prepared for on-chip SDS-PAGE per instructions from the supplier (heating to $65^{\circ} \mathrm{C}$ for 5 minutes). Prior to use, samples were diluted in tris/glycine/SDS run buffer by 
a factor of 3. The proteins $\alpha$-lactalbumin, trypsin inhibitor, carbonic anhydrase, and alcohol dehydrogenase were used as sizing standards for all gel concentrations in this work; aprotinin did not consistently provide sufficient signal-to-noise ratios to be used as a standard. Immunoassay results for the cytokine interleukin-2 (IL-2, $M_{r} 17.1 \mathrm{kDa}$ ) relied upon fluorescent labeling (FITC) of that species for detection.


Figure 3. (top) Schematic representing in situ photopolymerization process. Photopolymerization steps include: (a) microfluidic channels have been conditioned, (b) channels are filled with a solution of unpolymerized monomer and cross-linker, (c) select regions of the device are masked and the device is exposed to a UV light flood exposure, and (d) the final device contains channels filled with cross-linked polyacrylamide gel and open channels. (bottom) Inverted grayscale image of gel localized within the separation channel. For clarity, the gel has been dyed to appear dark in this image.

\section{RESULTS \& DISCUSSION}

On-chip SDS-PAGE. Directed experiments were conducted to assess the performance of on-chip SDS-PAGE in cross-linked gel sieving matrices. SDS-PAGE electropherograms are shown in Figure 4 for a mixture of five fluorescently labeled species for both $4 \%$ and $8 \%$ cross-linked gels. Under otherwise identical running conditions, the $4 \%$ gel is unable to resolve the species, while the $8 \%$ gel permits baseline resolution of all five species in elapsed separation times of $30 \mathrm{~s}$ or less. As an illustration of the exceptionally rapid SDS-PAGE possible with this technique, Figure 4 shows a near baseline-resolved separation that is complete in less than $5 \mathrm{~s}$. Separation lengths were typically less than $7 \mathrm{~mm}$. Conventional slab gel SDS-PAGE requires up to an hour for separation of species in gels having separation lanes $80 \mathrm{~mm}$ in length. Similarly, rapid near-baseline resolved on-chip protein separations were reliably achieved in cross-linked gels ranging from $6 \%$ to $12 \%$ total gel concentration.

Standard curves relating migration distance and molecular weight were generated to validate the separation mechanism governing the on-chip technique. Figure 5 shows an electropherogram corresponding to the SDS-PAGE separation from Figure 4 overlaid with a standard curve generated from that separation data. Standard curves were linear $\left(R^{2}>0.984\right)$ for gel concentrations ranging from $4 \%$ to $10 \%$. The linearity of the curves confirms the size-based separation mechanism of the technique, as is also the case in slab gel SDS-PAGE.



Figure 4. Time-evolution of an on-chip SDS-PAGE separation of protein in an $8 \%$ gel. Transverse band asymmetry is attributed to a non-optimal offset injection and low diffusion of the species. Cross-linked gel was patterned in all channels of the device. Species are: (1) apotinin (2) $\alpha$-lactalbumin, (3) trypsin inhibitor, (4) carbonic anhydrase II and (5) alcohol dehydrogenase. $E=$ $298 \mathrm{~V} / \mathrm{cm}$.



Figure 5. Standard curve overlaid with axial concentration distribution for the separation of four proteins in a $8 \%$ acrylamide SDS-PAGE separation. $E=298 \mathrm{~V} / \mathrm{cm}$.

The in situ photopatterning technique allows fabrication of various gel concentrations. Results for separations of $M_{r} 6.5$ to 39 $\mathrm{kDa}$ proteins using $4 \%$ and $8 \%$ gels are shown in Figure 6 . The results highlight the importance of tailoring gel concentration for optimum resolving power for a given assay (i.e. $M_{r}$ range of interest). Further, gel porosity customization enables development of quantitative system calibration curves employing Ferguson analysis. Protein standards were separated on gels having gel concentrations ranging from $4 \%$ to $10 \%$. The apparent mobility of each species was measured in those gel systems. Plotting logarithm mobility $v s$. gel concentration for each standard results in an effective retardation coefficient for each species. For the gel system reported in this work, a calibration curve relating this retardation coefficient to molecular weight was found to be governed by the equation: $K_{r}=2.2 \times 10^{-3} \times M_{r}-0.001\left(R^{2}=\right.$ 
0.996). Generation of such calibration curves based upon protein standards allows determination of $M_{r}$ for unknown proteins.

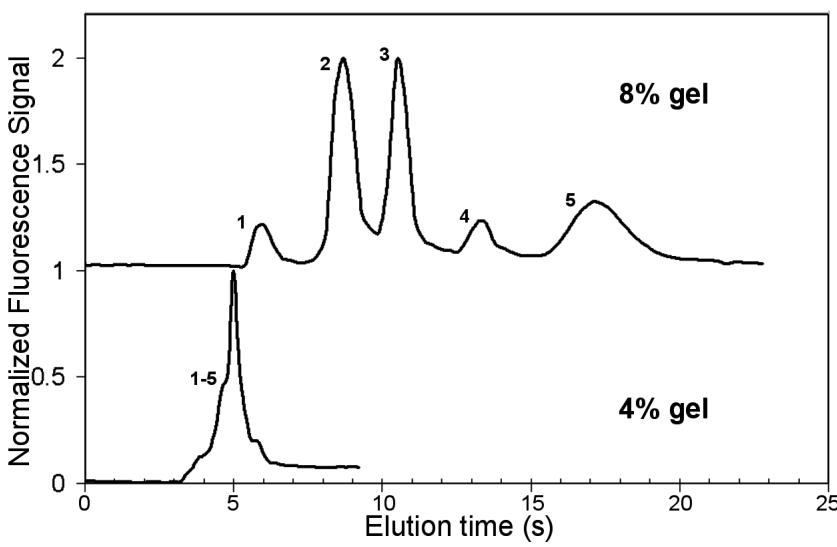

Figure 6. On-chip SDS-PAGE separation resolution dependence on gel acrylamide concentration (4\% and $8 \%$ ). Separation of five SDS-protein complexes was not attained in the low concentration gel, while the higher concentration gel clearly resolves all species. Aside from variation of gel concentration, conditions were constant. $E=298 \mathrm{~V} / \mathrm{cm}$.
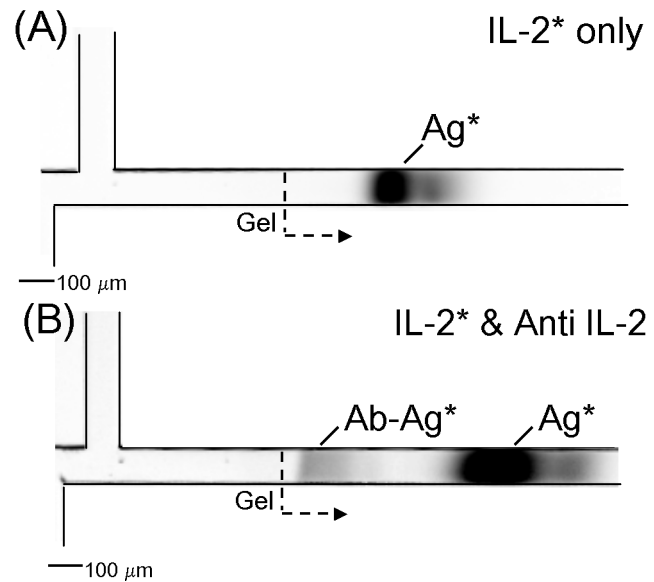

Figure 7. Native PAGE separation of free and immune-complexed IL-2. Inverted grayscale images of: (A) native PAGE of IL-2*, and $(B)$ native PAGE of the immune-complex with a $2 x$ molar excess of $I L-2 *$. Species were separated in less than $15 \mathrm{~s}$ and within $2 \mathrm{~mm}$ of the injector. $E=460 \mathrm{~V} / \mathrm{cm}$.

Immunoassays. Sensitive detection of cytokines is of interest to clinical diagnostics as such disease markers are involved in the immune response to invading species. Traditional immunoassays can be multi-step procedures, while electrophoretic immunoassays are rapid, sensitive, and amenable to automation. While not universally applicable, non-denaturing native PAGE can sometimes resolve free antigen from immune-complexed species without destroying immune complexes. Denaturing SDS-PAGE tends to disrupt immune complexes. Figure 7 shows on-chip immunoassays resulting in ultrafast native PAGE characterization of the cytokine interleukin 2 (IL-2). In these experiments, the antigen (Ag) was labeled used as a fluorescently labeled probe for the target antibody $(\mathrm{Ab})$. Figure $7 \mathrm{~A}$ shows native PAGE results for an IL-2* sample with no antibody present. A companion native PAGE analysis of IL-2* in solution with anti-IL-2 $\left(M_{r} 150 \mathrm{kDa}\right)$ is shown in shown Figure 7B. Figure 7 shows an additional, lower mobility band resolving from the antigen. The slower band corresponds to the large antigen-antibody immune-complex. Differentiation between the free IL-2* and the IL-2 immune complex occurred within $15 \mathrm{~s}$ in a $2 \mathrm{~mm}$ separation length.

\section{CONCLUSIONS}

Cross-linked polyacrylamide gels, photolithographically patterned in situ, have been demonstrated for use as components of microfluidic bioanalysis systems. The fabrication process is compatible with the microfluidic format and has been introduced as a means to define cross-linked sieving matrices at specific locations in a microfluidic device for use in protein sizing and electrophoretic immunoassay applications.

The fabrication technique has enabled direct adaptation of slab gel SDS-PAGE of proteins to a chip-based format, resulting in substantial performance improvements. Comparison of SDSPAGE on-chip to slab gel reveals separation times up to two orders of magnitude more rapid than those performed in gels $(30 \mathrm{~s} v \mathrm{~s}$. $3600 \mathrm{~s}$ ) and separation distances a fraction of those required for slab SDS-PAGE $(7 \mathrm{~mm}$ vs. $50 \mathrm{~mm})$. Further work extends the approach to separations of biomarkers, in this case IL-2 and the associated immune complex. Our lab is currently exploring this material as a component of an integrated diagnostic instrument.

\section{REFERENCES}

1. S. Mouradian, "Lab-on-a-chip: applications in proteomics" Current Opinions in Chemistry and Biology 6,1 (2002).

2. D. J. Harrison, et al., "Capillary Electrophoresis and Sample Injection Systems Integrated On a Planar Glass Chip" Anal. Chem. 64,17 (1992).

3. C. S. Effenhauser, A. Manz and H. M. Widmer, "Glass Chips For HighSpeed Capillary Electrophoresis Separations With Submicrometer Plate Heights" Anal. Chem. 65,19 (1993).

4. S. C. Jacobson, et al., "High-Speed Separations On a Microchip" Anal. Chem. 66,7 (1994).

5. A. T. Woolley and R. A. Mathies, "Ultra-High-Speed Dna-Sequencing Using Capillary Electrophoresis Chips" Anal. Chem. 67,20 (1995).

6. S. N. Brahmasandra, et al., "Electrophoresis in microfabricated devices using photopolymerized polyacrylamide gels and electrode-defined sample injection" Electrophoresis 22, (2001).

7. K. G. Olsen, D. J. Ross and M. J. Tarlov, "Immobilization of DNA hydrogel plugs in microfluidic channels" Anal. Chem. 15,74 (2002).

8. R. F. Probstein (1994). Physicochemical Hydrodynamics: An Introduction. New York, John Wiley \& Sons, INC.

9. A. Chrambach and D. Rodbard, "Polyacrylamide Gel Electrophoresis" Science 172, (1971).

10. A. L. Shapiro, E. Vinuela and J. V. Maizel, "Molecular Weight Estimation of Polypeptide Chains by Electrophoresis in SDSPolyacrylamide Gels" Biochem. Biophys. Res. Commun. 28, (1967).

11. W. S. B. Yeung, et al., "Capillary electrophoresis-based immunoassay" J. Chromatogr. B 25,797 (2003).

12. D. J. Throckmorton, T. J. Shepodd and A. K. Singh, "Electrochromatography in microchips: Reversed-phase separation of peptides and amino acids using photopatterned rigid polymer monoliths" Anal. Chem. 74,4 (2002).

13. B. J. Kirby, et al., "Programmable modification of cell adhesion and zeta potential in silica microchips" Lab Chip 3, (2003).

14. S. C. Jacobson, et al., "Effects of Injection Schemes and Column Geometry On the Performance of Microchip Electrophoresis Devices" Anal. Chem. 66,7 (1994).

\section{ACKNOWLEDGEMENTS}

This work was financially supported by a National Institute of Dental and Craniofacial Research grant (U01DE014961). Sandia is a multi-program laboratory operated by Sandia Corp., a Lockheed Martin Co., for the United States Department of Energy under Contract DE-AC0494AL85000. 